\title{
EFFICIENT DIRECT ORGANOGENESIS FROM SHOOT TIPS AND NODAL SEGMENTS OF ASH GOURD (BENINCASA HISPIDA L.)
}

\author{
M. Kausar, S. Parvin, M.E. Haque, M. Khalekuzzaman, B. Sikdar and M.A. Islam* \\ Professor Joarder DNA and Chromosome Research Lab. \\ Department of Genetic Engineering and Biotechnology \\ University of Rajshahi, Rajshahi 6205, Bangladesh \\ *Corresponding Author, e-mail: asadgen@ru.ac.bd
}

\begin{abstract}
The effect of external application of phytohormone on explants viz., shoot tips and nodal segments of ash gourd (Benincasa hispida L.) was tested on the frequency of shoot and root induction. Shoot tips and nodal segments were cultured on MS medium supplemented with different concentrations and combinations of cytokinins (BAP, Kinetin), auxin (IBA, NAA) and gibberellic acid $\left(\mathrm{GA}_{3}\right)$ for multiple shoot formation and root induction. The highest number (up to 90\%) of multiple shoot formation was obtained from the shoot tips in MS medium fortified with $1.5 \mathrm{mg} / \mathrm{l} \mathrm{BAP}+0.2 \mathrm{mg} / \mathrm{l} \mathrm{GA}_{3}$, where average number of shoots per culture was 5.55 . In case of nodal segment, better response (up to $78 \%$ ) for shoot multiplication was found in MS with $2.0 \mathrm{mg} / \mathrm{l} \mathrm{BAP}+0.2 \mathrm{mg} / \mathrm{l} \mathrm{GA}$. The concentration of $1.0 \mathrm{mg} / \mathrm{l} \mathrm{IBA}$ was found to be most effective for root initiation in microshoot developed from both types of explants. Rooted plantlets were acclimatatized and established in sandy soil with good survival rate.
\end{abstract}

Key words: Organogenesis, ash gourd, MS medium, shoot tips, nodal segments

সারাংশঃ চালকুমড়ার কাণ্ডশীর্ষ ও পর্বাংশ এক্সপ্লান্ট ব্যবহার করে কান্ডমুকুল ও মূল উৎপাদনে ফাইটোহরমোনের প্রয়োগের ব্যাহ্যিক প্রভাব পরীক্ষা করা হয়েছে। বহুমুকুল এবং মূল জন্মাবার উদ্দেশ্যে কাঞ্তশীর্ষ এবং পর্বাংশগুনো এমএস মিডিয়ানের সাথে বিভিন্ন ঘনত্বর ও মিশ্রণের সাইটোকাইনিন্স (বিএপি ও কাইনেটিন), অক্সিন (আইবিএ ও এনএএ) এবং জিব্রেলিক এসিড (জিএ•) সহযোগে কালচার করা হয়েছে। ১.৫ মিলিগ্রাম/লিটার বিএপি + ০.২ মিলিগ্রাম /লিটার জিএ• হরনোনগুলি এমএস মিডিয়ানের মধ্যে ব্যবহার করে কাঞ্তশীর্ষ থেকে সবচেয়ে বেশি (৯০\% পর্যন্ত) বহু-কান্ডমুকুল উৎপাদন সম্ভব হয়েছে এবং যেখানে কালচার প্রতি মুকুল সংখ্যা দাঁড়িয়েছে ৫.৫৫। পর্বাংতের ক্ষেত্রে কান্ডমুকুল উৎপাদনের জন্য ২.০ মিলিগ্রাম/লিটার বিএপি + ০.২ মিলিগ্রাম/লিটার জিএ• ব্যবহার করে ভাল ফল পাওয়া গেছে (৭৮\% পর্যন্ত)। উভয় ধরনের এক্সপ্লান্টের ক্ষেত্রে ১.০ মিলিগ্রাম/ লিটার ঘনত্বের আইবিএ ব্যবহার করে ক্ষুদ্র কান্ডমুকুল থেকে সর্বাধিক মূল উৎপাদন পরিলক্ষিত হয়েছে। শিকড় গজানো অণুচারাগুনোকে বালুযুক্ত মাটিতে খাপ খাওয়ানো এবং স্থায়ীকরণ করা হয়েছে। এদের বেঁঁে থাকার হার যথেষ্ট ভালো।

\section{Introduction}

Ash gourd or wax gourd (Benincasa hispida L.) is a monocieous vegetable. Ash gourd is belonging to the family cucurbitaceae. The Curbitaceae family is a tropical and botanically highly specialized family among the climbing plants. Ash gourd is a popular vegetable in Bangladesh, India and neighboring countries. An immature and mature fruit of ash gourd is used in curries. The immature fruit is called fuzzy melons which have a delicate taste and flavour. In India, they are used extensively in curries. The fruits are valued for its medicinal properties. In India and China, they are used as an anthelmintic, antiperiodic and aphrodisiac for lowering blood sugar, against epilepsy, insanity and other nervous diseases. They are recommended in Ayurvedic medicine for the management of peptic ulcers. The seeds are used as a verminfuge. Ash gourd or winter melon is a nutritive fruit that grows from a vine. It has a long shelf life and can be stored for up to 12 months. The plants mainly propagated from seed. In vitro culture is used to some degree in the improvement of almost every major agronomic, vegetable and fiber crop species (Cade et al. 1987). Micropropagation is an alternative way to get quality seedlings in the crop. In vitro multiplication of elite clones will be an attractive approach in order to meet the requirement of quality propagules at large scale for commercial cultivation (Kumar et al. 2003). The improvement of ash gourd can be done by local breeding system or by genetic transformation system. Genetic transformation is a reliable method for development of plants by specific gene transfer (Sanford and Johnston 1985). Successful genetic transformation system requires a reliable and efficient regeneration system. Hence, the present work was designed to develop an efficient protocol for in vitro regeneration of ash gourd which could be used further in genetic transformation system for its development.

\section{Materials and Methods}

The work was carried out at Professor Joarder DNA and Chromosome Research Laboratory, Department of Genetic Engineering and Biotechnology, University of Rajshahi, Bangladesh. Shoot tips and nodal segments were collected from mature healthy in vivo grown plants of Benincasa hispida and the explants were washed thoroughly in running tap water for 15 min. Then these were treated with $1 \%$ Tween- 80 for eight minutes and again washing with distilled water to make the materials free from Tween-80. Further sterilization was done under aseptic condition in a laminar airflow cabinet. Surface sterilization was carried out with $0.05 \% \mathrm{HgCl}_{2}$ for 2 minutes by gentle shaking and 
rinsed 4-5 times in sterilized doubled distilled water to remove $\mathrm{HgCl}_{2}$. Explants were cut into $1 \mathrm{~cm}$ in length and transferred in culture tube with $15 \mathrm{ml}$ agar semisolified with 1.0\% Difco Bacto agar MS (Murashige and Skoog, 1962) medium. The medium was supplemented with 3\% sucrose and different hormonal concentrations BAP (0.5-3 mg/l), GA 3 (0.1-0.2mg/l), Kinetin (0.1-0.2mg/l), IBA (0.5-2.0 mg/l), NAA ((0.1$0.2 \mathrm{mg} / \mathrm{l}$ and $0.5-2.0 \mathrm{mg} / \mathrm{l}$ ) for direct organogenesis. $\mathrm{pH}$ of the medium was adjusted to 5.7 and was autoclaved by stream sterilization at $121^{\circ} \mathrm{C}$ for $22 \mathrm{~min}$ at 1.0 $\mathrm{kg} / \mathrm{cm}^{3}$ pressure. The cultures were maintained at $25 \pm$ $2^{\circ} \mathrm{C}$ under the cool white fluorescent tubes at 3000 lux with a photoperiod of 16 hours. The shoot formation was checked by 3 times of culture in same medium. Then in vitro developed shoots were subcultured in root induction medium with different concentrations of IBA and NAA. After development of sufficient roots, the micro plantlets were considered ready to transfer in soil. The roots of the plantlets are gently washed by distilled water to remove agar attached to the root zone. Immediately these were transferred to small pots containing a mixture of sand, soil and humus in the ratio of 1:2:2 for acclimatization. The newly transplanted plantlets were covered by polythene paper and kept in growth chamber for 7 days. Polythene covering was removed and the plantlets were transferred into the larger pot containing soil. Each treatment had 10 replicates of culture and experiments were repeated twice.

\section{Results and Discussion}

To develop a suitable protocol for axillary shoot development of ash gourd, two types of explants, shoot tips and nodal segments were cultured on MS medium with different concentrations and combinations of phytohormone viz., BAP, $\mathrm{GA}_{3}, \mathrm{Kn}$ and NAA. It was observed that the effect of $\mathrm{GA}_{3}$ with BAP was better for shoot multiplication than either the effect of BAP singly or combination of $\mathrm{Kn}$ and NAA (Figure 1A-C). It has been reported that the typeof explants and combinations of shoot induction media are effective for further shoot induction(Sarkeret al.2003).In a significant development of suitable protocol for shoot multiplication, we observed shoot tips explants gave better results than nodal segments (Table-1). When MS medium was fortified with $1.5 \mathrm{mg} / \mathrm{l} \mathrm{BAP}+0.2 \mathrm{mg} / \mathrm{l} \mathrm{GA}_{3}, 90 \%$ shoot tip explants developed multiple shoot and the mean number of shoot formation was $5.55 \pm 0.55$, but in case of nodal segment, only $78 \%$ explants produced shoots on the same combination of BAP and $\mathrm{GA}_{3}$. Best response for multiple shoot regeneration was obtained by Haque et al. (2008) from the nodal segments of Benincasa hispida on MS $+1.50 \mathrm{mg} / \mathrm{l}$ BAP and they observed the frequency of multiple shoot formation from nodal segments was 90\%. However, only BAP was also found to be effective for in vitro shoot multiplication in other species such as Alpinia purpurata by Chang \& Criley, (1993), Cucumis melo by Chan \& Lok (2005), Momordica charantia by Agarwal \& Kamal (2004) and in Langenaria siceraria by Han et al. (2004). Huda and Sikdar (2006) reported that MS medium fortified with the combinations of BAP, IBA, $\mathrm{GA}_{3}$ was very effective for shoot initiation and elongation from apical meristem of bitter gourd. In this study, it was found that low concentration of cytokinin enhanced shoot multiplication. In other species many researchers reported the similar results (Kathal et al.1988, 1994; Sing et al. 1996 in Cucumis melo and Haque et al. 2010 in pumpkin).

Root induction was carried out by placing individual microshoots on $1 / 2$ MS medium containing various concentrations of NAA and IBA (Figure 1D\&E). Efficient rooting was achieved on $1 / 2$ MS medium supplemented with $1.0 \mathrm{mg} / \mathrm{l}$ IBA and 90\% microshoots were produced root. Whereas, $70 \%$ microshoots were responsed with the application of NAA (Table 2). Our results were supported by Kathiravan et al. (2006). Haque et al. (2008) reported root induction of ash gourd using $1.5 \mathrm{mg} / \mathrm{l}$ IBA. Debnath et al. (2000) were achieved efficient rooting using half strength MS + $0.5 \mathrm{mg} / \mathrm{l}$ NAA in Trichosanthes dioica. The potential of in vitro propagated ash gourd plantlets were used for ex-vitro establishment. The plantlets were transferred in soil in pots after 3 weeks of initial hardening under culture-room conditions. Almost $70 \%$ of these regenerate plantlets survived and showed development of new branches (Figure 1F).

In this investigation, several conditions of tissue culture were optimized for the culture and micropropagation of ash gourd. These include selection of explants, combination and concentration of Plant Growth Regulators (RGPs) for multiple shoot induction, elongation of induced shoots favoured by root induction and proliferation and eventually successful transplantation of plantlets in in vivo condition.

Micropropagation of ash gourd has been achieved through in vitro culture of nodal segments and shoot tips as explants. The technique described appears to be readily adaptable for large scale micro propagation of ash guard. 
Table 1 Effect of different concentrations and combinations of auxin, cytokinin and gibberellic acid on multiple shoots formation from shoot tips and nodal segments

\begin{tabular}{|c|c|c|c|c|c|c|}
\hline \multirow{2}{*}{$\begin{array}{l}\text { Growth } \\
\text { regulators } \\
(\mathrm{mg} / \mathrm{l})\end{array}$} & \multicolumn{3}{|c|}{ Nodal segment } & \multicolumn{3}{|c|}{ Shoot tip } \\
\hline & $\begin{array}{l}\text { \% of explant } \\
\text { responded }\end{array}$ & $\begin{array}{l}\text { Mean no. of shoot } \\
\text { per culture } \\
(\bar{X} \pm S E)\end{array}$ & $\begin{array}{c}\begin{array}{c}\text { Mean length of } \\
\text { the shoot(cm) } \\
(\bar{X} \pm S E)\end{array} \\
\end{array}$ & $\begin{array}{l}\text { \% of explant } \\
\text { responded }\end{array}$ & $\begin{array}{l}\text { Mean no. of shoot } \\
\text { per culture } \\
(\bar{X} \pm S E)\end{array}$ & $\begin{array}{c}\begin{array}{c}\text { Mean length of } \\
\text { the shoot }(\mathbf{c m}) \\
(\bar{X} \pm S E)\end{array} \\
\end{array}$ \\
\hline \multicolumn{7}{|c|}{ BAP } \\
\hline 0.5 & - & - & - & - & - & - \\
\hline 1.0 & 60 & $3.00 \pm 0.656$ & $3.10 \pm 0.24$ & 60 & $3.10 \pm 0.05$ & $3.00 \pm 0.21$ \\
\hline 1.5 & 65 & $3.50 \pm 0.88$ & $3.60 \pm 0.22$ & 65 & $4.30 \pm 0.11$ & $3.45 \pm 0.33$ \\
\hline 2.0 & 65 & $4.10 \pm 0.57$ & $4.10 \pm 0.09$ & 80 & $\mathbf{5 . 5 0} \pm 0.70$ & $\mathbf{5 . 8 0} \pm 0.18$ \\
\hline 2.5 & 70 & $5.00 \pm 0.99$ & $\mathbf{5 . 0 0} \pm 0.25$ & 75 & $4.40 \pm 0.44$ & $4.50 \pm 0.19$ \\
\hline 3.0 & 30 & $3.50 \pm 0.33$ & $3.20 \pm 0.86$ & 30 & $3.68 \pm 0.15$ & $3.40 \pm 0.04$ \\
\hline \multicolumn{7}{|c|}{$\mathrm{BAP}+\mathrm{GA}_{3}$} \\
\hline $0.5+0.1$ & - & - & - & - & - & - \\
\hline $0.5+0.2$ & 50 & $3.10 \pm 0.12$ & $3.38 \pm 0.06$ & 60 & $3.10 \pm 0.22$ & $3.40 \pm 0.15$ \\
\hline $1.0+0.2$ & 60 & $3.60 \pm 0.55$ & $4.18 \pm 0.12$ & 65 & $4.38 \pm 0.18$ & $3.85 \pm 0.08$ \\
\hline $1.5+0.2$ & 80 & $5.18 \pm 1.12$ & $5.88 \pm 0.85$ & 90 & $\mathbf{5 . 5 5} \pm 0.55$ & $6.00 \pm 0.02$ \\
\hline $2.0+0.2$ & 70 & $4.81 \pm 0.42$ & $4.85 \pm 0.42$ & 75 & $4.80 \pm 0.09$ & $4.50 \pm 0.11$ \\
\hline $2.5+0.2$ & 55 & $3.88 \pm 0.18$ & $3.50 \pm 0.18$ & 70 & $3.80 \pm 0.13$ & $3.80 \pm 0.23$ \\
\hline $3.0+0.2$ & 20 & $3.00 \pm 0.33$ & $3.1 \pm 0.09$ & 40 & $3.2 \pm 0.25$ & $3.0 \pm 0.44$ \\
\hline \multicolumn{7}{|c|}{$\mathrm{BAP}+\mathrm{Kn}$} \\
\hline $0.5+0.1$ & - & - & - & - & - & - \\
\hline $0.5+0.2$ & 35 & $1.08 \pm 0.12$ & $1.70 \pm 0.06$ & 20 & $1.00 \pm 0.22$ & $3.00 \pm 0.15$ \\
\hline $1.0+0.2$ & 40 & $1.50 \pm 0.05$ & $1.44 \pm 0.52$ & 35 & $1.81 \pm 0.05$ & $3.45 \pm 0.06$ \\
\hline $1.5+0.2$ & 50 & $\mathbf{4 . 0 3} \pm 0.52$ & $\mathbf{5 . 1 0} \pm 0.13$ & 40 & $3.16 \pm 0.18$ & $2.50 \pm 0.11$ \\
\hline $2.0+0.2$ & 45 & $3.10 \pm 0.04$ & $4.00 \pm 0.22$ & 45 & $5.80 \pm 0.20$ & $3.38 \pm 0.25$ \\
\hline $2.5+0.2$ & 30 & $3.00 \pm 0.33$ & $3.50 \pm 0.09$ & 20 & $3.00 \pm 0.09$ & $2.50 \pm 0.03$ \\
\hline $3.0+0.2$ & 15 & $2.30 \pm 0.07$ & $2.22 \pm 0.50$ & 15 & $1.10 \pm 0.16$ & $2.38 \pm 0.22$ \\
\hline \multicolumn{7}{|c|}{$\mathrm{BAP}+\mathrm{NAA}$} \\
\hline $0.5+0.1$ & - & - & - & - & - & - \\
\hline $0.5+0.2$ & 35 & $2.00 \pm 0.44$ & $2.55 \pm 0.05$ & 30 & $3.10 \pm 0.08$ & $0.90 \pm 0.13$ \\
\hline $1.0+0.2$ & 55 & $2.30 \pm 0.25$ & $3.00 \pm 0.22$ & 50 & $4.38 \pm 0.12$ & $1.54 \pm 0.05$ \\
\hline $1.5+0.2$ & 60 & $3.60 \pm 0.08$ & $4.00 \pm 0.12$ & 65 & $3.88 \pm 0.16$ & $3.80 \pm 0.25$ \\
\hline $2.0+0.2$ & 65 & $4.15 \pm 0.18$ & $4.88 \pm 0.55$ & 70 & $\mathbf{5 . 0 0} \pm 0.21$ & $4.50 \pm 0.10$ \\
\hline $2.5+0.2$ & 45 & $2.10 \pm 0.11$ & $3.10 \pm 0.28$ & 65 & $3.00 \pm 0.09$ & $3.10 \pm 0.33$ \\
\hline $3.0+0.2$ & 25 & $1.90 \pm 0.27$ & $1.60 \pm 0.33$ & 40 & $2.16 \pm 0.11$ & $1.50 \pm 0.02$ \\
\hline
\end{tabular}

Table 2 In vitro root induction in regenerated shoots

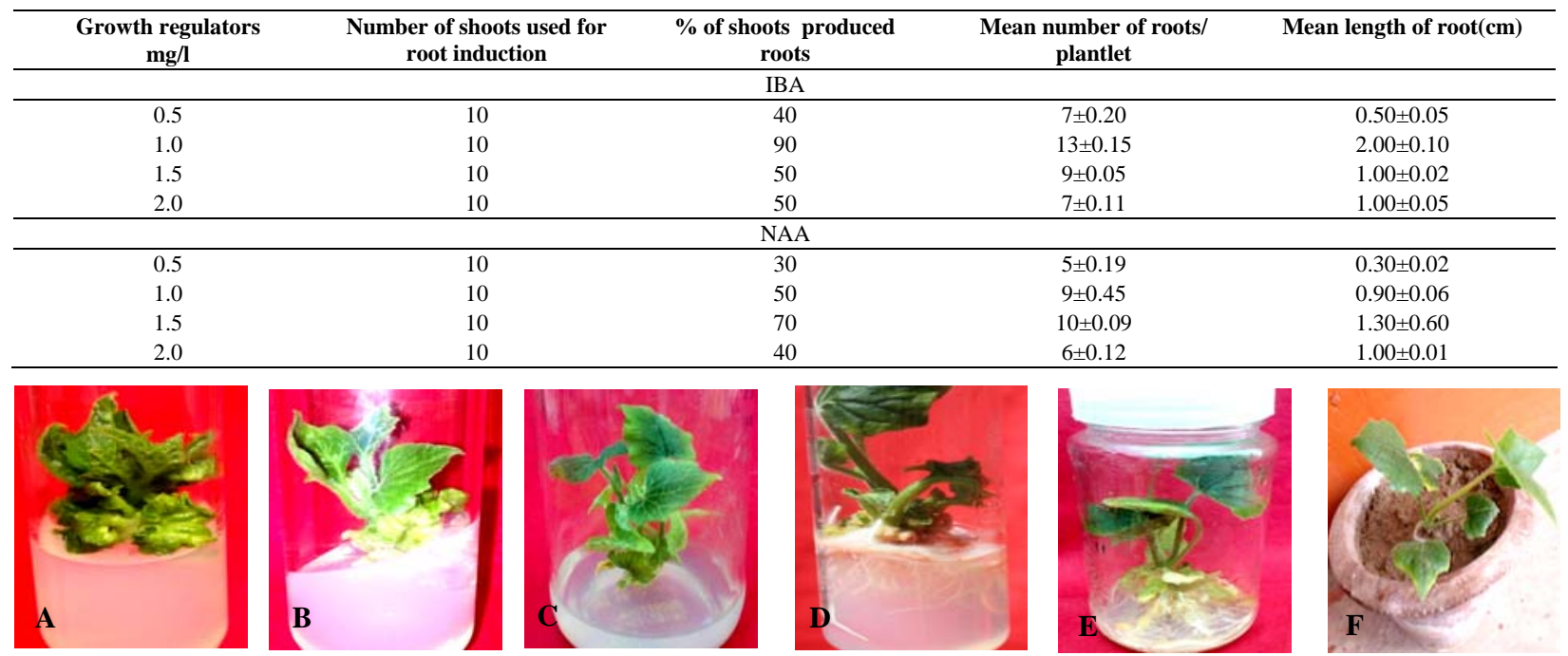

Fig. 1A. Multiple shoot proliferation from shoot tip explants on MS $+1.5 \mathrm{mg} / \mathrm{l} \mathrm{BAP}+0.2 \mathrm{mg} / \mathrm{l} \mathrm{GA}$

B. Multiple shoot proliferation from nodal segment explants on MS $+2.5 \mathrm{mg} / \mathrm{l}$ BAP

C. Multiple shoot proliferation from nodal segment explants on MS $+1.5 \mathrm{mg} / \mathrm{l} \mathrm{BAP}+0.2 \mathrm{mg} / \mathrm{l} \mathrm{GA}_{3}$

D \& E. Root formation on $1 / 2 \mathrm{MS}+1.0 \mathrm{mg} / \mathrm{l} \mathrm{IBA}$ and $1 / 2 \mathrm{MS}+1.5 \mathrm{mg} / \mathrm{l} \mathrm{NAA}$

F. In vitro raised plantlets grown under ex vitro environment 
Acknowledgements: The authors gratefully acknowledge USDA and MoE, Government of the People's Republic of Bangladesh for the financial supports.

\section{References}

Agarwal M and Kamal R. 2004. In vitro clonal propagation of Momordica charentia L. Indian J. Biotech. 3:426-430.

Cade RM, Wehner TC and Blazich FA. 1987. Organogenesis and embryogenesis from cucumber (Cucumis sativus L.) cotyledon derived callus. Hort. Sci. 22:1130 (Abstr).

Chan LK and Lok KH. 2005. In vitro plantlets regeneration from nodal segments of musk melon. Biotechnology, 4(4): 354-357.

Chang BKW and Criley A. 1993. Clonal propagation of pinc ginger in vitro. Hort. Sci. 28: 1203.

Debnath RK, Roy SK, Ahmed G and Hossain M. 2000. Micropropagation of Patal (Tricosantes dioica Roxb.) from nodal segment and shoot tip. Plant tiss. cult. 10(2): 125-130.

Han JS, Oh DG, Mok IG, Park HG, Kim CK. 2004. Efficient plant regeneration from cotyledon explants of bottle gourd (Lagenaria siceraria Standl.). Plant Cell Rep. 23: 291-296.

Haque ME, Sarker MAR, Mahmud MA, Rezwana D and Sikdar B. 2008. In vitro propagation of pumpkin and ash guard through nodal segments. J. Bio. Sci. 16: 67-71.

Haque ME, Rezwana D, Islam MA, Sikdar B. 2010. In vitro regeneration of pumpkin (Cucurbita maxima) through shoot apical meristem. J. Bio. Sci. 18: 104-107.
Huda AKMN and Sikdar B. 2006. In vitro plant production through apical meristem culture of Bitter gourd (Momordica charantia L.). Plant Tiss. Cult. Biotech. 16(1): 31-36.

Kumar HGA, Murthy HN and Murthy KY and Paek KY. 2003. Embryogenesis and plant regeneration from anther cultures of Cucumis sativus L. Sci. Hortic. 98:213-222.

Kathiravan K, Vengedesan G, Singer S, Steinitz B, Paris H and Gaba V. 2006. Adventitious regeneration in vitro occurs across a wide spectrum of squash (Cucurbita pepo) genotypes. Plant Cell Tiss. Org. Cult. 85: 285-295.

Kathal R, Bhatnagar SP and Bhojwani SS. 1988. Regeneration of plants from leaf explant of Cucumis melo cv. pusasharbati. Plant Cell Rep, 7: 449-451.

Kathal R, Bhatnagar SP and Bhojwani SS. 1994. Plant regeneration from the callus derived root from explants of Cucumis melo L. cv. pusa sharbati Rekha. Plant Sci. 96: 137-142.

Murashig T and Skoog F. 1962. A reveised medium for rapid growth and bioassay with tobacco tissue culture. Physi. Plant 15: 473497.

Sandford JC and Johnston SA. 1985. The concept of parasite desired resistance deriving resistance genes from the parasite's own genome. J. Theor. Biol. 113: 395-405.

Sarker RH, Mustafa BM, Biswas A, Mahbub S, Nahar M, Hasem R and Hoque MI. 2003. In vitro regeneration in lentil (Lens culinaris Medik.) Plant Tissue Cult. 13: 155-163.

Singh MN, Mishra AK and Bhatnagar SP. 1996. In vitro production of plants from cotyledon explant of Cucumis melo L. and their successful transfer to field. Phytomorph. 46: 395-402.

Manuscript received on 9 September 2013 and revised on 16 November 2013 